It has been assumed here that $O$ lies within the parabola so that $b<a$. When $O$ is without the parabola, the equation will be

$$
r_{1} . O F_{2}-r_{2} . O F_{1} \pm r . F_{1} F_{2} \sqrt{\frac{X+b}{\bar{X}+2 b-a}}=0,
$$

and, if $2 a$ be the angle between the two tangents drawn from 0 to the given parabola, $\cos 2 a=\frac{X+a}{a-2 b-X}, 2 \sin ^{2} a=\frac{2(X+b)}{X+2 b-a}$,

or the vector equation is

$$
r_{1} . O F_{2}-r_{1} . O F_{1} \pm r . F_{1} F_{2} \sin \alpha=0 .
$$

When $O$ lies on the parabola, $b=a, O F_{1}=0$, or $F_{1}$ cuincides with $O$, which is then a triple focus (and cusp) of the pedal, $k_{1}=0, k_{2}=-2$, and the coordinates of $F_{2}$, the only real single focus, will be $X-4 a,-Y$; $O F$, being normal at $F$, to the parabola $y^{2}=4 a(x+4 a)$. When $O$ lies on the axis, on the opposite side of the focus to the verter, $b=0$, $O F_{1}, O F_{2}^{\prime}$ are normals drawn from $O$ to the parabola $y^{2}=4 a(x+a)$, and the vector equation is $r_{1}+r_{2}=2 r$.

\title{
On the Calculation of Symmetric Functions.
}

$$
\begin{gathered}
\text { By. Mr. J. Hammond, M.A. } \\
\text { [Read January 12th, 1882.] }
\end{gathered}
$$

1. Consider the equation

$$
(x-\mu)\left(x^{n}-p_{1} x^{n-1}+p_{2} x^{n-2}-p_{3} x^{n-3}+\ldots\right)=0 \text {. }
$$

suppose the roots of the equation of the $n^{\text {th }}$ degree to be $a, \beta, \gamma, \ldots$, and let

$$
\Sigma a^{q} \beta^{q} \ldots \gamma^{r} \delta^{4} \ldots . . .=\left(p_{1}, p_{2}, p_{2}, \ldots . . .\right) ;
$$

the introduction of the new root $\mu$, on the one hand, changes $p_{1}, p_{2}, p_{3}, \ldots$ into $p_{1}+\mu, p_{2}+\mu p_{1}, p_{8}+\mu p_{2}, \ldots$, and, on the other, introduces into the symmetrio functiou new terms of the form

Hence

$$
\mu^{2} \Sigma a^{2} \ldots \gamma^{r} \delta^{2} \ldots \ldots .
$$

$$
\begin{aligned}
& \Sigma a^{q} \beta^{q} \ldots \gamma^{r} \delta^{q} \ldots \ldots+\mu^{q} \Sigma a^{q} \ldots \gamma^{r} \delta^{q} \ldots \ldots+\mu^{r} \Sigma u^{q} \beta^{q} \ldots \delta^{q} \ldots \ldots+\mu^{r} \Sigma u^{q} \beta^{q} \ldots \gamma^{r} \ldots \ldots \\
& =\phi\left(p_{1}+\mu, p_{1}+\mu p_{1}, p_{3}+\mu p_{2}, \ldots \ldots\right)
\end{aligned}
$$

If now the expanded value of $\phi$, in (2), is written

$$
\phi+\mu \phi_{1}+\mu^{2} \phi_{2}+\mu^{3} \phi_{2}+\ldots \ldots,
$$


equating coefficients, we have

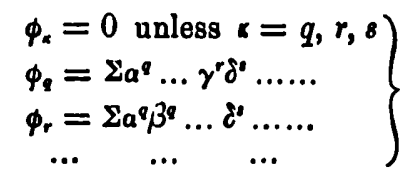

Or, the symmetric fanction equivalent to $\phi_{\alpha}$ is obtained by simply cancelling one of the letters in the original symmetric function that occurs with the inder $x$. If we wish to find the value of $\Sigma a^{n}$,

and $\phi_{n}=1$.

$$
\phi_{1}, \phi_{2}, \ldots \phi_{n-1}=0 \text {, }
$$

2. Several terms of a symmetric function can often be found by inspection from known formulø.

Thus, let

$$
\phi=\Sigma a^{q} \beta^{r} \gamma^{2} \ldots . . .,
$$

and suppose $\Sigma_{m} n^{q} \beta^{r} \gamma^{2} \ldots$ to denote the value of the symmetric function when $p_{m+1}$ and all succeeding coefficients in (1) are equated to zero; then $\phi\left(p_{1}, p_{3}, p_{3}, \ldots p_{n}\right)=\phi\left(p_{1}, p_{2}, p_{8}, \ldots p_{m}, 0,0, \ldots\right)$ t terms containing $p_{m+1}, p_{m+2}, p_{m+3} \ldots p_{n}$ $=\Sigma_{m} a^{p} \beta^{r} \gamma^{*} \ldots . . .+$ otber terms

In the case $\Sigma a^{5} \beta^{4} \gamma^{2} \delta^{2} e$, we have

$$
\Sigma_{6} a^{8} \beta^{4} \gamma^{8} \delta^{2} \varepsilon=p_{8} \Sigma_{8} a^{4} \beta^{8} \gamma^{2} \delta
$$

and, referring to tables of the $10^{\text {th }}$ degree, we see that

$$
\begin{aligned}
& \Sigma \mathrm{u}^{8} \beta^{4} \gamma^{2} \delta^{2} e=p_{6}\left(p_{1} p_{3} p_{3} p_{4}-3 p_{8}^{2} p_{4}-3 p_{1}^{2} p_{4}^{2}+4 p_{2} p_{4}^{2}-3 p_{1} p_{2}^{2} p_{8}+4 p_{1}^{2} p_{8} p_{6}\right. \\
& \left.+5 p_{8} p_{8} p_{8}-5 p_{6}^{2}\right) \\
& + \text { terms containing } p_{0}, p_{7}, p_{8}, \ldots p_{15} \text {. }
\end{aligned}
$$

If $\phi=\Sigma a^{n}$, it is easily seen that

$$
\begin{aligned}
\Sigma_{3} a^{n}=p_{1}-n p_{1}^{n-2} p_{2} & +\frac{n(n-3)}{2 !} p_{1}^{n-4} p_{2}^{2}-\frac{n(n-4)(n-5)}{3 !} p_{1}^{n-1} p_{2}^{3} \\
& +\frac{n(n-5)(n-6)(n-7)}{4 !} p_{1}^{n-8} p_{2}^{4}-\& c .
\end{aligned}
$$

In fact, if we consider the equation

$$
x^{2}-2 x \cos \theta+1=0,
$$

in which $p_{1}=2 \cos \theta$ and $p_{2}=1$, it is obvions that $\Sigma_{2} a^{n}=2 \cos n \theta$, and hence that the coefficients in (5) are those of the development of $2 \cos n \theta$ in descending powers of $2 \cos \theta$.

This gives $\frac{n+1}{2}$ or $\frac{n}{2}+1$ of the leading terms of $\mathrm{\Sigma a}^{n}$ according as $n$ is odd or oren. 
3. The use of $\phi_{1}$ alone will sometimes enable us to calculate the value of a symmetric function, and in the case where $\Sigma$ contains no letters with unity for their index, the determination will be independent of the values of symmetrio functions of inferior degrees, since here $\phi_{1}=0$.

For example, if $\phi=\Sigma a^{2} \beta^{2} \gamma^{2} \ldots . .$, the index of the last letter being 1 or 2 according as $n$ is odd or even.

First, wheu $n=2 m$, assume

$$
\begin{array}{r}
\phi=p_{m}^{2}+A_{1} p_{m-1} p_{m+1}+A_{2} p_{m-2} p_{m+2}+A_{3} p_{m-3} p_{m-2}+\ldots \\
\ldots+A_{m-1} p_{1} p_{2 m-1}+A_{m} p_{2 m} ;
\end{array}
$$

operating with

$$
\frac{d}{d p_{1}}+p_{1} \frac{d}{d p_{2}}+p_{3} \frac{d}{d p_{3}}+\ldots \ldots
$$

we have

$$
\begin{array}{r}
\phi_{1}=p_{m-1} p_{m}\left(2+A_{1}\right)+p_{m-2} p_{m+1}\left(A_{1}+A_{2}\right)+p_{m-3} p_{m+2}\left(A_{2}+\Delta_{3}\right)+\ldots \\
\ldots+p_{2 m-1}\left(A_{m-1}+A_{m}\right),
\end{array}
$$

where, since in this case $\phi_{1}=0$, it follows that

$$
\begin{gathered}
A_{1}=-2, A_{2}=2, \ldots \ldots A_{m}=(-)^{m} 2, \\
\text { i.e., } \quad \sum a^{2} \rho^{2} \gamma^{2} \ldots=p_{m}^{2}-2 p_{m-1} p_{m+1}+2 p_{m-2} p_{m+2}-\ldots+(-)^{m} 2 p_{2 m}
\end{gathered}
$$

Secondly, if $n=2 m+1$, assume

$$
\phi=p_{m} p_{m+1}+B_{1} p_{m-1} p_{m+2}+B_{2} p_{m-2} p_{m+3}+\ldots+B_{m-1} p_{1} p_{2 m}+B_{m} p_{1 m+1} \text {; }
$$

as before,

$\phi_{1}=p_{m}^{2}+p_{m-1} p_{m+1}\left(1+B_{1}\right)+\dot{p}_{m-2} p_{m+2}\left(B_{1}+B_{2}\right)+\ldots+\left(B_{m-1}+B_{m}\right) p_{2 m ;}$

but here, since the index of the last. letter in $\dot{\Sigma}$ is nuity, this value of $\phi_{1}$ must be equivalent to (6). Hence

$$
1+B_{1} \doteq-2, \quad B_{1}+B_{1}=2, \ldots \ldots B_{m-1}+B_{m}=(-)^{m} 2,
$$

giving $\Sigma \alpha^{2} \beta^{2} \gamma^{2} \ldots=p_{m} p_{m+1}-3 p_{m-1} p_{m+2}+5 p_{m-2} p_{m+2}-\ldots$

$$
\ldots+(-)^{m}(2 m+1) p_{2 m+1}
$$

Again, if $\phi=\Sigma a^{2} \beta \gamma \delta \ldots=u_{n}$, the destruction of the last letter changes $u_{n}$ into $u_{n-1}$, so that, if

$$
\begin{aligned}
& \qquad u_{n}=p_{1} p_{n-1}+A_{n} p_{n}, \\
& \qquad \begin{array}{l}
\left(\frac{d}{d p_{1}}+p_{n-2} \frac{d}{d p_{n-1}}+p_{n-1} \frac{d}{d p_{n}}\right) u_{n}=u_{n-1}=p_{1} p_{n-2}+\left(1+A_{n}\right) p_{n-1} \\
1+A_{n}=A_{n-1}
\end{array} \\
& \text { From this we obtain }
\end{aligned}
$$

$$
\text { and finally } \quad \Sigma a^{2} \beta \gamma \delta \ldots=p_{1} p_{n-1}-\imath p_{n}
$$

4. But $\phi_{1}$, which is of weight $n-1$, will not in many cases give p.10ugh relations among the coefficients of $\phi$, whioh is of weight $n$, to vol. xIII.-No. 185. 
determine all of them. In snch cases the missing relations can be supplied by asing single terms of $\phi_{2}, \phi_{3}, \ldots$; the method of finding suoh terms is in every case the same as in the following example, wherv $n=11$, and it is required to find the coefficient of $p_{1} p_{2} p_{2}^{2}$ in $\phi_{2}$.

If we consider all the factors of $p_{1} p_{2} p_{\mathrm{a}}^{2}$, not exceeding in this case the second degree, viz., $1, p_{1}, p_{2}, p_{2}, p_{1} p_{2}, p_{1} p_{3}, p_{2} p_{3} p_{3}^{2}$, and the corresponding terms of the operator, in this case

$$
\frac{1}{2 !}\left(\frac{d^{2}}{d p_{1}^{2}}+2 p_{1} \frac{d^{2}}{d p_{1} d p_{2}}+\ldots\right) \text {; }
$$

we see that the term $p_{1} p_{1} p_{2}^{2}$ in $\phi_{9}$ can only come from

$$
\begin{aligned}
& \frac{1}{2} \frac{d^{2}}{d p_{1}^{2}}\left(p_{1}^{3} p_{3} p_{3}^{2}\right), \quad p_{1} \frac{d^{2}}{d p_{1} d p_{2}}\left(p_{1} p_{2}^{2} p_{3}^{2}\right), \quad p_{2} \frac{d^{2}}{d p_{1} d p_{3}}\left(p_{1}^{2} p_{3}^{2}\right), \\
& p_{2} \frac{d^{2}}{d p_{1} d p_{4}}\left(p_{1}^{2} p_{3} p_{3} p_{4}\right), \quad p_{1} p_{2} \frac{d^{2}}{d p_{3} d p_{3}}\left(p_{2} p_{3}^{3}\right), \quad p_{1} p_{2} \frac{d^{2}}{d p_{3} d p_{4}}\left(p_{2}^{2} p_{3} p_{3}\right) \text {, } \\
& p_{3} p_{3} \frac{d^{2}}{d p_{2} d p_{4}}\left(p_{1} p_{3}^{2} p_{4}\right), \quad \frac{1}{t} p_{3}^{2} \frac{d^{2}}{d p_{4}^{2}}\left(p_{1} p_{2} p_{4}^{2}\right) ;
\end{aligned}
$$

and hence, if

$$
\begin{aligned}
& \phi=\ldots \ldots+A p_{1}^{3} p_{3} p_{2}^{2}+B p_{1} p_{2}^{2} p_{3}^{2}+O p_{1}^{2} p_{3}^{3}+D_{p_{1}^{2}} p_{2} p_{s} p_{\mathrm{s}} \\
& +E p_{2} p_{3}^{2}+F p_{2}^{2} p_{8} p_{4}+G p_{1} p_{3}^{2} p_{4}+H p_{1} p_{3} p_{4}^{2}+\ldots \ldots . . \\
& \phi_{2}=\ldots \ldots+(3 A+2 B+6 C+2 D+3 E+2 F+2 G+H) p_{1} p_{2} p_{3}^{2}+\ldots \ldots .
\end{aligned}
$$

and if we have already found all bat one of the quantities $A, B, \ldots H$, the remaining ons is found from $\phi_{9}$.

The remaining article consists of applications of this principle, chiefly to the case $\Sigma a^{n}$.

5. Suppose

$$
\begin{aligned}
\phi=A_{0} p_{1}^{n}+A_{2} p_{1}^{n-2} p_{8}+A_{2} p_{1}^{n-3} p_{3}+\ldots \\
\ldots+A_{n-2} p_{1}^{2} p_{n-3}+A_{n-1} p_{1} p_{n-1}+A_{n} p_{n}+\text { other terms, }
\end{aligned}
$$

then, if $k<n$ the last terms of $\phi_{1}, \phi_{2}, \ldots \phi_{a}$ are respectively

$$
\left(A_{n-1}+A_{n}\right) p_{n-1}, \quad\left(A_{n-3}+A_{n-1}\right) p_{n-2}, \ldots\left(A_{n-\ldots}+A_{n-a+1}\right) p_{n-4} ;
$$

for the factors of $p_{n-a}$ are simply 1 and $p_{n-n}$, so that the last term of $\phi_{a}$ is obtained from

$$
\frac{1}{\kappa !} \frac{d^{*}}{d p_{1}^{*}}\left(A_{n-\infty} p_{i}^{*} p_{n-a}\right),
$$

and

$$
\frac{1}{(\kappa-1) !} p_{n-\alpha} \frac{d^{*}}{d p_{1}^{n-1} d p_{n-\alpha+1}}\left(A_{n-\alpha+1} p_{1}^{\alpha-1} p_{n-\alpha+1}\right),
$$

and is therefore

$$
\left(A_{n-a}+A_{n-\varepsilon+1}\right) p_{n-\ldots} \text {. }
$$


When $\phi=\Sigma a^{n}$, since from (5), or otherwise, we have $A_{1}=-n$, it follows that $\quad A_{8}=n, A_{4}=-n, \ldots A_{n}=(-)^{n+1} n$.

But if $\phi=\Sigma a^{q} \beta^{r}$, where $q>r$ and $q+r=n$, we have

for other values of $x$; thus

$$
\phi_{r}=\Sigma a^{q}, \quad \phi_{q}=\Sigma a^{r}, \quad \phi_{k}=0,
$$

$$
A_{q}+A_{q+1}=\text { coeff. } p_{q} \text { in } \Sigma a^{q}=(-)^{q+1} q,
$$

and

$$
A_{r}+A_{r+1}=(-)^{r+1} r \text {. }
$$

Here, then,

$$
A_{2}, A_{3}, \ldots A_{r}=0 \text {, }
$$

$$
A_{r+1}=(-)^{r+1} r, A_{r+2}=(-)^{r+2} r, \ldots \ldots A_{4}=(-)^{r} r,
$$

$A_{q+1}=(-)^{q+1}(q+r)=(-)^{q+1} n, A_{q+2}=(-)^{q+2} n, \ldots \ldots A_{n}=(-)^{n} n$.

These values must, however, be modified in the case

$$
q=r=\frac{n}{2}
$$

when

$$
A_{q+1}=(-)^{q+1} \frac{n}{2}, \ldots \ldots A_{n}=(-)^{n} \frac{n}{2} .
$$

Other symmetric functions may be similarly treated.

Again, suppose

$$
\phi=\ldots \ldots+A_{m} p_{1}^{n-m} p_{m}+B_{m} p_{1}^{n-m-2} p_{s} p_{m}+A_{m+1} p_{1}^{n-m-1} p_{m+1}+\ldots \ldots .,
$$

and consider the term $p_{1}^{n-m-1} p_{m}$ in $\phi_{1}$; facturs $1, p_{1}, p_{m}$.

This term is

$$
\begin{gathered}
\frac{d}{d p_{1}}\left(A_{m} p_{1}^{n-m} p_{m}\right)+p_{1} \frac{d}{d p_{2}}\left(B_{m} p_{1}^{n-m-2} p_{s} p_{m}\right)+p_{m} \frac{\dot{d}}{d p_{m+1}}\left(A_{m+1} p_{1}^{n-m-1} p_{m+1}\right) \\
=\left\{(n-m) A_{m}+B_{m}+A_{m+1}\right\} p_{1}^{n-m-1} p_{m}, \text { if } m>2 .
\end{gathered}
$$

When $m=2$,

$$
\begin{aligned}
& \phi=\ldots \ldots+A_{2} p_{1}^{n-2} p_{2}+B_{2} p_{1}^{n-4} p_{2}^{3}+A_{2} p_{1}^{n-3} p_{2}+\ldots \ldots, \\
& \text { and } \quad \phi_{1}=\ldots \ldots+\left\{(n-2) A_{2}+2 B_{2}+A_{3}\right\} p_{1}^{n-3} p_{2}+\ldots \ldots .
\end{aligned}
$$

Hence $A_{2}, A_{3}, \ldots A_{n}$ having been found from the last terms of $\phi_{1}, \phi_{2}, \ldots ; B_{2}, B_{8}, \ldots$, can be found from $\phi_{1}$ only.

If $\phi=\Sigma a^{n}$, since

$$
A_{2}=-n, \ldots A_{m}=(-)^{m+1} n_{1}
$$

it follows that

$$
B_{2}=\frac{n(n-3)}{2} \text {, }
$$

which agrees with (5), and

$$
\begin{gathered}
B_{m}=(-)^{m} n\left(\begin{array}{c}
n-m-1) \\
\circ 2
\end{array}\right. \\
02
\end{gathered}
$$


the last of the series being

$$
B_{n-2}=(-)^{n} n=\text { coeff. } p_{2} p_{n-2} \text { in } \Sigma a^{n}, n>4 \text {. }
$$

If, as before, $m>2$, the term $p_{1}^{n-m-8} p_{m}$ in $\phi_{2}$, whose factors are $1, p_{1}$, $p_{m}, p_{1}^{2}, p_{1} p_{m}$, can only originate from the five terins

$$
\begin{gathered}
\frac{1}{d p_{1}^{2}}\left(p_{1}^{n-m} p_{m}\right), \quad p_{1} \frac{d^{3}}{d p_{1} d p_{2}}\left(p_{1}^{n-m-2} p_{2} p_{m}\right), \\
p_{m} \frac{d^{2}}{d p_{1} d p_{m+1}}\left(p_{1}^{n-m-1} p_{m+1}\right), \quad \frac{p_{1}^{2}}{2} \frac{d^{3}}{d p_{2}^{2}}\left(p_{1}^{n-m-1} p_{2}^{2} p_{m}\right),
\end{gathered}
$$

and

$$
p_{1} p_{m} \frac{d^{3}}{d p_{2} d p_{m+1}}\left(p_{1}^{n-m-3} p_{2} p_{m+1}\right) ;
$$

Bo that with coefficients $A_{m} \ldots B_{m} \ldots$ as before, if the coefficient of ${y_{1}^{n-m-1}}_{p_{2}^{2}}^{n} p_{m}$ in $\phi$ be denoted by $\left(I_{m}\right.$, the term in $\phi_{2}$ is

$$
\left\{\begin{aligned}
\frac{(n-m)(n-m-1)}{2} A_{m}+(n-m-2) B_{m}+(n-m-1) A_{m+1} & \\
& +O_{m}+B_{m+1}
\end{aligned}\right\} p_{1}^{n-m-2} p_{m},
$$

whence the coefficients $C_{m}$ are determined.

In the case $\Sigma a^{n}$, substituting for the $A^{\prime}$ 's and $B^{\prime}$ s their valnes found above, we obtain

$$
C_{m}=(-)^{m+1} \frac{n(n-m-2)(n-m-3)}{2}, m>2
$$

the last term of the series being

$$
\sigma_{n-1}=(-)^{n+1} n \text {. }
$$

The calculation of $C_{2}$, like that of $B_{2}$, will only reproduce a term of (5). Coefficients of other terms of $\mathrm{\Sigma a}^{n}$ may be found in the same way, without the slightest variation in the method.

Thursday, February 9 th, 1882.

S. ROBERTS, Esq., F.R.S., President, in the Chair.

Mr. J. H. Tompson, F.C.S., F.G.S., Science Master in the Auckland College, New Zealand, was elected a Member, and Mrs. Bryant was admitted into the Society.

Mr. Tucker read a short abstract of a paper by Mr. H. M. Jeffery, F.R.S., "On certain Quartic Curves having a Tacnode at Infinity at which the Line at Infinity is the Multiple Tangent." 
Mr. J.W. L. Glaisher, F.R.S., having taken the Chair pro tem., the President communicated some results connected with Euler's Formula connecting the sum of the Divisors of a Number with the Pentagonal Numbers, and remarked that the formnla really expressed the equality of the sum of the divisors to the sam of the $m^{\text {ta }}$ powers of the roots of a certain equation.

Mr. Hammond and Mr. Tucker also made brief communications.

The following presents were received :-

Carte-de-visite likeness from Mr. C. E. Bickmore.

"Mémoires de la Société des Sciences Physiques et Naturelles de Bordeaux," 2 Série, Tome iv., 30 Cahier ; Parie, 1881.

"Atti della R. Accademia dei Lincei-Transunti," Vol. vi., Faso. $3^{\circ}, 4^{\circ}, 6^{\circ}$.

" Educational Times," February, 1882.

"Proceedings of Royal Society," Vol. xxxiii., No. 217.

“ Tidsskrift for Mathematik . . . . Fjerde Røkke, Feunte Aargang, Forste, Andet, Tredie, Fjerde, Femte, Sjette Hefte ;" Kjфbenhavn, 1881.

"Archires Néerlandaises des Sciences Exactes et Naturelles," Tome xvi., 3me, $4^{\mathrm{me}}, 5^{\text {me }}$ Livraivons; Harlem, 1881.

"Jahrbuch über die Fortschritte der Mathematik," elfter Band, Heft 3 ; Jahrgang 1879 ; Berlin, 1882.

Donations to the Bodleian Library during the year ending November 8, 1881 ; Oxford, 1881.

"The Scientific Proceedings of the Royal Dublin Snciety," Vol. ii., Pt. vii. (November, 1880), Vol. iii., Pt. i. (January, 1881), Pt. ii. (April, 1881), Pt. iii. (July, 1881), Pt. iv. (Octcber, 1881).

"The Scientific Transactious of the Royal Dublin Snciety," Vol. i., Series ii., Pt. xiii., "On the Possibility of Originating Wuve Disturbances in the Ether by means of Electric Forces," Pt. 2, by G. F. Fitzgerald (read May 19th, 1880). Pt. xiv., "Explorations in the Bone Cave of Ballynumintra, near Capprgb, County Waterford," by A. L. AJams, G. H. Kinuhan, and R. J. Ussher (read November 15th, 1880).

The following three Mémoires (des xii. Bundes der "Abhandlungen der Muthematisch.Physischen Classe der Königl-sächsischen Gesellschaft der Wissenschafton"):-

C. Neumann: "Ubur dit Peripolaren Coordinaten," November (Leiprig, 1880).

C. Neumann: "Die Vertheilung der Elektricität auf einer Kugelcalùtto," November 1 (Leipzig, 1880).

W. G. Hankel: “ Elektrische Untersuchungen, fünfzehnte Abhandlung über dio aktino- und l'iezo-elektrischen Eigenschaften di's Bergkrystalles und ihre Bezichung zu den Thermo-elektrischen," No. vii. (Leipzig, 1881). 